\title{
Genetische Beziehungen für das Merkmal Lebenstagszunahme in einem Dreirassenkreuzungsprogramm beim Schwein
}

\begin{abstract}
Summary
Title of the paper: Genetic association for daily gain (lifetime) in an crossbreeding program

The aim of this investigation is an analysis of the crossing structure of a three-race crossing program and the genetic relations with respect to the trait average daily gain. For this purpose we examine the genetic relations for their usefulness for a estimation of genetic parameters and breeding values. Finally a parameter estimation is carried out.

The data basis consists of 1757 German Large White performances, 34980 Landrace performances and 2775 Pietrain performances in pure breed as well as 92757 performances of the crossings of the mother lines and altogether 13854 Pietrain crossings (1997 PI x DL and 11857 PI x DEDL).

The heritabilities for the variable daily gain are in the range from 0.17 to 0.31 . The genetic correlations between the mother races and their crossbreedings are around 0.9. Between the pure Pietrain and the Pietrain crossings these values reach only 0.7 . Within the two groups of crossings with Pietrains the genetic correlations are almost one.

From the genetic point of view it is possible to put together the mother races and their crossings as well as the Pietrain crossings. Thus, for this trait it is sufficient to consider a three variable model with separate investigation of the mother races, the Pietrains and the Pietrain crossings.
\end{abstract}

Key Words: genetic correlation, crossbred, daily gain, pig

\section{Zusammenfassung}

Ziel der Untersuchung war eine Analyse der Kreuzungsstruktur eines Dreirassenkreuzungsprogrammes und deren genetischer Beziehungen für das Merkmal Lebenstagszunahme. Dazu wurden die genetischen Verknüpfungen auf ihre Brauchbarkeit für eine Parameter- und Zuchtwertschätzung untersucht und dargestellt sowie eine Parameterschätzung durchgeführt.

Als Datengrundlage wurden 1757 Edelschwein-, 34980 Landrasse- und 2775 Pietrainleistungen in Reinzucht sowie 92757 Leistungen der Kreuzungen der Mutterlinien und insgesamt 13854 Pietrainanpaarungen (1997 PI x DL und 11857 PI x DEDL) ausgewertet.

Die Heritabilitäten für das Merkmal Lebenstagszunahme erreichten Werte von 0,17 bis 0,31. Die genetischen Korrelationen zwischen den Mutterrassen und deren Kreuzungsprodukten lagen bei 0,9. Zwischen den reinen Pietrain und den Pietrainanpaarungen erreichten diese nur Werte von 0,7. Innerhalb der beiden Kreuzungsgruppen mit Pietrainanteil waren die genetischen Korrelationen fast eins.

Aus genetischer Sicht ist eine Zusammenfassung der Mutterrassen und deren Kreuzungen sowie der Pietrainkreuzungen möglich. Somit ist für dieses Merkmal ein Dreimerkmalsmodell mit der jeweils separaten Betrachtung der Leistungen der Mutterrassen, der Pietrain und der Pietrainkreuzungen ausreichend.

Schlüsselwörter: Genetische Korrelationen, Kreuzung, Lebenstagszunahme, Schwein

1. Einleitung

Die Kreuzungszucht ist die bevorzugte Methode zur Erzeugung der Endprodukte in der Schweinezucht. Damit werden besonders zwei Ziele verfolgt, zum einen in der Nutzung von Heterosiseffekten (z.B. Fruchtbarkeit oder Umweltstabilität) in den Nachkommen und zum anderen in der Umgehung ungünstiger genetischer 
Zusammenhänge zwischen einzelnen Leistungsmerkmalen (z.B. Mastleistung und Schlachtkörperwert), welche über die Kombination der Elternlinien vermindert werden können.

Damit spielt die simultane Verwendung von Reinzucht- und Kreuzungsleistungen in der Zuchtwertschätzung und somit auch in der Varianzkomponentenschätzung eine zentrale Rolle (siehe auch RÖHE u.a., 2000). Das zunehmende Interesse beruht dabei unter anderem auf folgenden Punkten:

- Größerer Materialumfang und damit steigende Genauigkeit auch für die Zuchtwerte der Reinzuchttiere (Informationsgewinn)

- $\quad$ Ausrichtung des Zuchtzieles auf die Gebrauchstiere (KRIETER, 1992; WEI, 1992)

- $\quad$ Große wirtschaftliche Bedeutung der Kreuzungsleistungen

- $\quad$ Notwendigkeit einer Betrachtung als unterschiedliche Merkmale, wenn die genetischen Korrelationen zwischen Reinzucht und Kreuzung deutlich von eins abweichen

Die kombinierte Nutzung von Reinzucht- und Kreuzungsleistungen erfordert insbesondere die Kenntnis der genetischen Parameter der Ausgangspopulationen und der Kreuzungsprodukte. Dabei haben die genetischen Korrelationen zwischen Reinzucht- und Kreuzungsleistungen einen besonderen Stellenwert.

An einem sächsischen Datenmaterial wurden die genetischen Beziehungen in einer Dreirassenkreuzung untersucht. Für die genetischen Verknüpfungen konnten die ausreichend dokumentierten Leistungen aus einem Vermarktungsverbund sowie zusätzlich noch Reinzuchtinformationen aus der Eigenleistungsprüfung auf Station sowie dem Feldtest der Sauen einbezogen werden.

Ziel der Untersuchung war eine Analyse der Kreuzungsstruktur in Sachsen sowie eine Aussage, inwieweit die Nutzung der Reinzuchtinformationen auch eine Verbesserung der Kreuzungsleistungen erwarten lässt, und ob die Leistungen in Reinzucht und Kreuzung als genetisch unterschiedlich determinierte Merkmale zu betrachten sind.

2.

Material und Methode

2.1

Datenmaterial

Als Datenmaterial standen folgende Informationsquellen der Geburtsjahre 1988 bis 1998 zur Verfügung:

- $\quad$ Ergebnisse des Feldtestes der Endstufeneber (nur auf bestimmten Betrieben getestet)

- $\quad$ Eigenleistungsergebnisse der Sauen aus der Feldprüfung

- $\quad$ Leistungsprüfanstalt-Daten

In die Untersuchung konnten aufgrund des vorliegenden Materialumfanges der Kreuzungstiere folgende genotypische Konstruktionen einbezogen werden:

- $\quad$ Deutsche Landrasse (DL)

- Deutsches Edelschwein (DE)

- $\quad$ Pietrain (PI)

- $\quad$ Kreuzungen aus Edelschweineber mit Landrassesauen (DEDL)

- Kreuzungen aus Pietrainebern mit den DEDL-Sauen (PIDEDL)

- $\quad$ Kreuzungen aus Pietrainebern mit Landrassesauen (PIDL) 
Der Schwerpunkt liegt hierbei auf den Informationen aus der typischen Dreirassenkreuzung (PI x DEDL). Die Kreuzungsleistungen von Tieren der Kombination PIDL sollen mit einbezogen werden, um eine Aussage über eine möglichen Zusammenfassung der Leistungen aller Kreuzungsprodukte von Pietrainebern zu erzielen.

Als Merkmal wurde die Lebenstagszunahme untersucht, welche im vorliegenden Material den größten Umfang aufwies.

Statistische Maßzahlen der Lebenstagszunahme sind im folgenden aufgeführt. Dabei wurden die Plausibilitätsgrenzen der gegenwärtigen Zuchtwertschätzung (300 1100 g) mit 292 g für die Endprodukte der Dreirassenkreuzung leicht unterschritten. Diese geringe Abweichung sollte jedoch keinen Einfluss auf die Ergebnisse haben.

Tabelle 1

Anzahl, Minimum, Maximum, Mittelwert und Standardabweichung für das Merkmal Lebenstagszunahme für die einbezogenen Genotypen (Data size, minimum, maximum, mean and standard deviation for daily gain sorted by genotypes)

\begin{tabular}{lrrrrr}
\hline Genotyp & Anzahl & min & max & $\bar{x}$ & s \\
\hline DE & 1757 & 376 & 901 & 613,72 & 79,28 \\
DL & 34980 & 323 & 928 & 550,13 & 50,42 \\
DEDL & 92757 & 316 & 969 & 569,77 & 50,35 \\
PI & 2775 & 318 & 871 & 578,65 & 85,18 \\
PIDL & 1997 & 316 & 751 & 535,75 & 57,76 \\
PIDEDL & 11857 & 292 & 848 & 545,74 & 61,88 \\
\hline
\end{tabular}

In den Rassen Deutsches Edelschwein und Pietrain sind, entsprechend der Stellung dieser Rassen im Kreuzungszuchtprogramm, die geringsten Anzahl Datensätze zu verzeichnen. Bei den Kreuzungsprodukten spielt die Kombination Pietraineber mit Landrassesau die geringste Bedeutung bei den einbezogenen Kreuzungskombinationen. Dennoch soll auch diese mit untersucht werden, da immer noch mehr Leistungsinformationen als für das Deutsche Edelschwein vorliegen. Hieran kann auch eine mögliche Zusammenfassung der Pietrainkreuzungsprodukte untersucht werden.

Die Verteilung der Leistungen über die Geschlechter zeigt nahezu nur weibliche $\mathrm{F}_{1}$ Tiere (DEDL) und natürlich keine oder fast keine Börge für die Ausgangsrassen sowie keine Eberleistungen bei den Kreuzungsprodukten. Somit sind entsprechende Geschlechtseffekte für diese genotypischen Konstruktionen nicht schätzbar.

\subsection{Datenverknüpfung}

Für die Schätzung genetischer Parameter in einem Kreuzungsprogramm sind neben tiefer liegenden genetischen Verknüpfungen über entsprechende Großväter o.ä. folgende Informationen besonders wertvoll:

- Eltern von Kreuzungstieren, welche eine Eigenleistung im untersuchten Merkmal (also Reinzuchtleistung) besitzen und somit eine Verknüpfung Reinzucht - Kreuzung gegeben ist, sowie

- Eltern, welche sowohl Nachkommen in Reinzucht und Kreuzung aufweisen. Die Qualität dieser Informationsquellen ist für eine günstige Struktur zur Ermittlung der Covarianzen zwischen den genotypischen Konstruktionen mit entscheidend.

In den folgenden beiden Abbildungen sind die Anzahl Datensätze bzw. Eltern in den entsprechenden Kreisen bzw. Quadraten dargestellt. Um die Größenverhältnisse zu veranschaulichen entsprechen die Kreisdurchmesser, die Diagonalen der Quadrate 
sowie die Strichstärke der Pfeile dem Logarithmus zur Basis 10 der jeweiligen Anzahl. Im oberen Bereich sind jeweils die Reinzuchtleistungen dargestellt, darunter die Kreuzungsleistungen.

In der Abbildung 1 ist in den Quadraten die Gesamtanzahl DE und PI-Eber sowie DLSauen dargestellt, die im Material vorkommen. Die nichtschraffierten Kreise (mit der Anzahl Datensätze) repräsentieren hier die absolute Anzahl Leistungsinformationen in den entsprechenden Genotypen, während die schraffierten Kreise den Anteil der Leistungen darstellen, welche auf Eltern mit einer Eigenleistung zurückzuführen sind. Die Überlappung dieser Anteile in den Kreuzungsleistungen ist ein Maß für den Anteil dieser Tiere, welche sowohl von einer eigenleistungsgeprüften Sau wie auch eines eigenleistungsgeprüften Vaters abstammen.

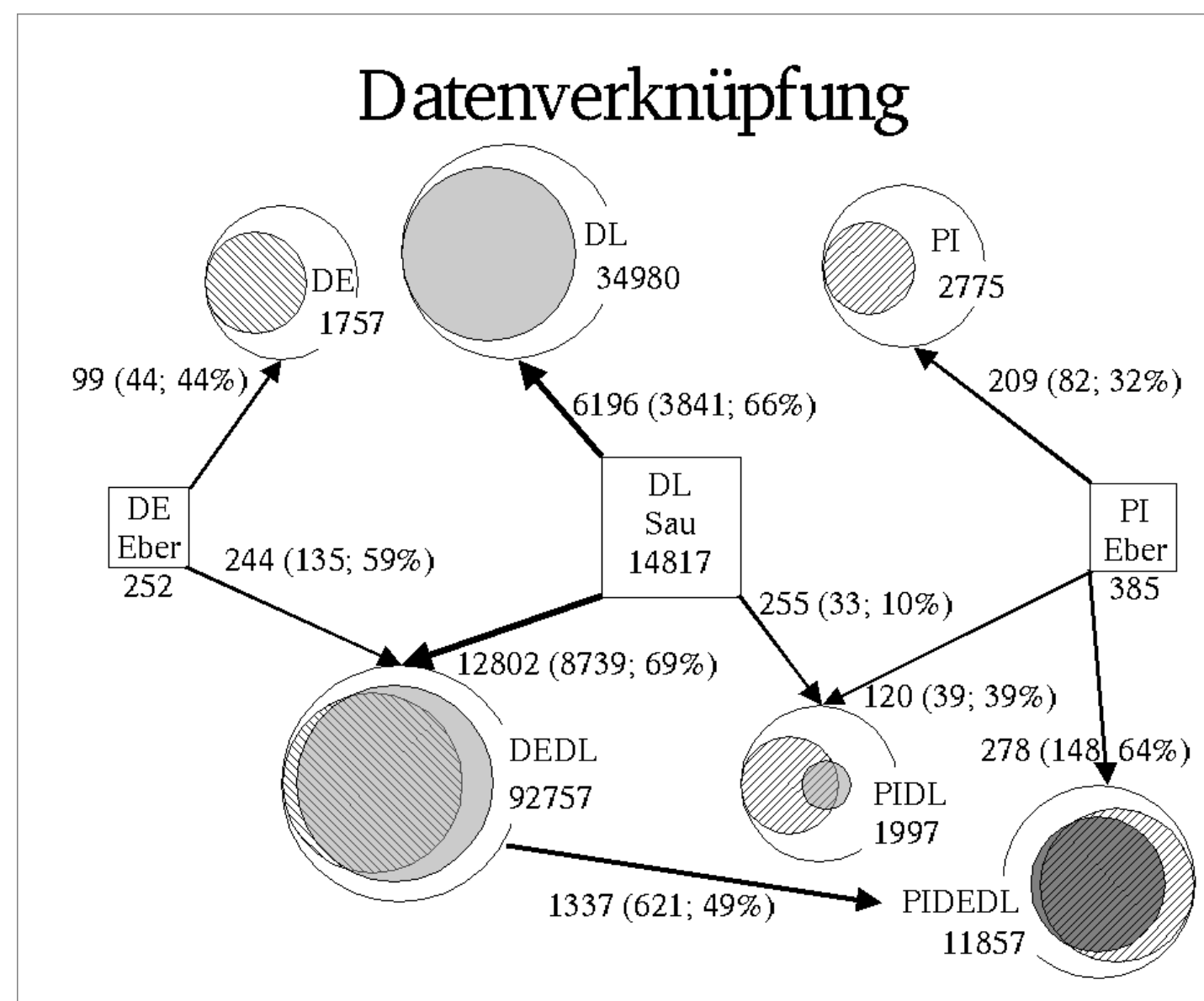

Beiträge von Deutsches Edelschwein $\bigcirc$ Deutsche Landrasse $\oslash$ Pietrain

Abb. 1: Anzahl Datensätze sowie Eltern für die verschiedenen genotypischen Konstruktionen sowie die Anzahl Eigenleistungen mit dem jeweiligen Anteil an Datensätzen der durch diese erklärt wird (Data size and number of parents with counted daily gain and part of offsprings that has this animals as parents)

An den Pfeilen ist die Anzahl der Eltern für den entsprechenden Genotyp dargestellt, dahinter in Klammern die Anzahl Eltern mit Eigenleistung und der prozentuale Anteil an der Gesamtdatenmenge, welchen diese Eigenleistungstiere zu direkten Vorfahren haben. Dieser Anteil ist dann noch einmal in Form der schraffierten Kreisen dargestellt und repräsentiert diesen Anteil an der Gesamtdatenmenge.

Insgesamt sind 252 Edelschweineber im Material vorhanden. 99 Eber kommen als Reinzuchtväter und 244 Eber in der Kreuzung vor. Von letzteren können 135 Eber 
eine Eigenleistung aufweisen. Diese sind Väter von 59 \% aller Kreuzungstiere dieser Kombination. Diese Tiere können also in der Varianzkomponenten- oder Zuchtwertschätzung einen direkten Bezug zwischen Reinzucht- und Kreuzungsleistung vermitteln. Die Tiere mit einer Eigenleistung und Anpaarung in Reinzucht sind für die genetische Verknüpfung zwischen Reinzucht- und Kreuzung nicht weiter von Bedeutung, so dass auf diese Informationen nicht gesondert eingegangen wird. Dennoch sind diese Informationen für die Schätzung der Reinzuchtparameter von enormer Bedeutung, welche hier aber nicht im Mittelpunkt stehen. Von den 8739 in Kreuzung angepaarten Landrassesauen mit Eigenleistung stammen sogar $69 \%$ der Kreuzungstiere ab. 85 \% der Datensätze, die von einer eigenleistungsgeprüften Mutter stammen, haben auch einen eigenleistungsgeprüften Vater (Überlappung der beiden schraffierten Flächen).

Von den 209 Pietrainvätern, welche in Reinzucht eingesetzt wurden, haben nur 82 auch eine Eigenleistung aufzuweisen. Dies spiegelt den großen Anteil Importeber wider, die dann unter den sächsischen Bedingungen keine Eigenleistung aufweisen können. Pietraineber mit Eigenleistung erklären in den beiden Kreuzungskombinationen 39 \% bzw. 64 \% der Datensätze der Nachkommen. Diese Anteile sind größer als in Reinzucht und verdeutlichen, dass für die Erzeugung der Mastendprodukte eher selbst gezogene Eber im Einsatz sind. DL-Sauen, die für die Kreuzungskombination PIDL als Mütter auftreten, sind nur vereinzelt mit einer Eigenleistung vorhanden. Diese sind auch nur von $10 \%$ aller Datensätze dieser genotypischen Konstruktion die Mütter. Bei den eigentlichen Endstufenprodukten (PIDEDL) sind 1337 Kreuzungssauen als Mütter zu verzeichnen. Von diesen können 621 eine Eigenleistung aufweisen, welche die Mütter von 49 \% aller Endprodukte sind.

In Abbildung 2 sind die Eltern aufgeführt, welche Nachkommen in beiden jeweils mit Pfeilen verbundenen Genotypen aufweisen können. Ansonsten gelten die für die vorherige Abbildung gemachten Bemerkungen. Somit haben 91 der insgesamt 99 (siehe Abb. 1) in Reinzucht vertretenen Edelschweineber auch Nachkommen in Kreuzung. Diese können $70 \%$ der Kreuzungsleistungen erklären und fast alle Reinzuchtleistungen (97\%).

Durch Landrassesauen werden $37 \%$ der Kreuzungsleistungen repräsentiert. In der Kreuzungskombination Pietrain mit Landrasse (PIDL) gibt es nur eine Mutter, welche sowohl Nachkommen in Reinzucht wie auch in dieser Kombination aufweisen kann. Die Pietraineber erklären für diese Kombination 41 \% der Leistungsdatensätze. Fast genauso groß (42\%) ist der Anteil, den Pi-Eber als Vater an den Endprodukten (PIDEDL) haben, welche auch in Reinzucht genutzt wurden. Dabei kommen alle Väter von PIDL-Tieren, die in Reinzucht Nachkommen haben, auch in der Kombination PIDEDL vor (also 46 Eber, alle in den 102 Ebern enthalten; Überlappung der beiden rechts schräg schraffierten Kreise). 118 Eber, von insgesamt 120 Vätern in PIDL, haben Nachkommen sowohl in der Anpaarung mit DL-Sauen als auch in der Kombination mit $\mathrm{F}_{1}$-Sauen.

Insgesamt sind die vorhandenen Strukturen für eine Parameter- bzw. Zuchtwertschätzung als günstig zu beurteilen. Eine routinemäßige Umsetzung würde jedoch eine breitere Dokumentation der Abstammung der Mastendprodukte erfordern, welche im Moment nur über eine Vermarktungskette realisiert werden konnte. 


\section{Datenverknüpfung}

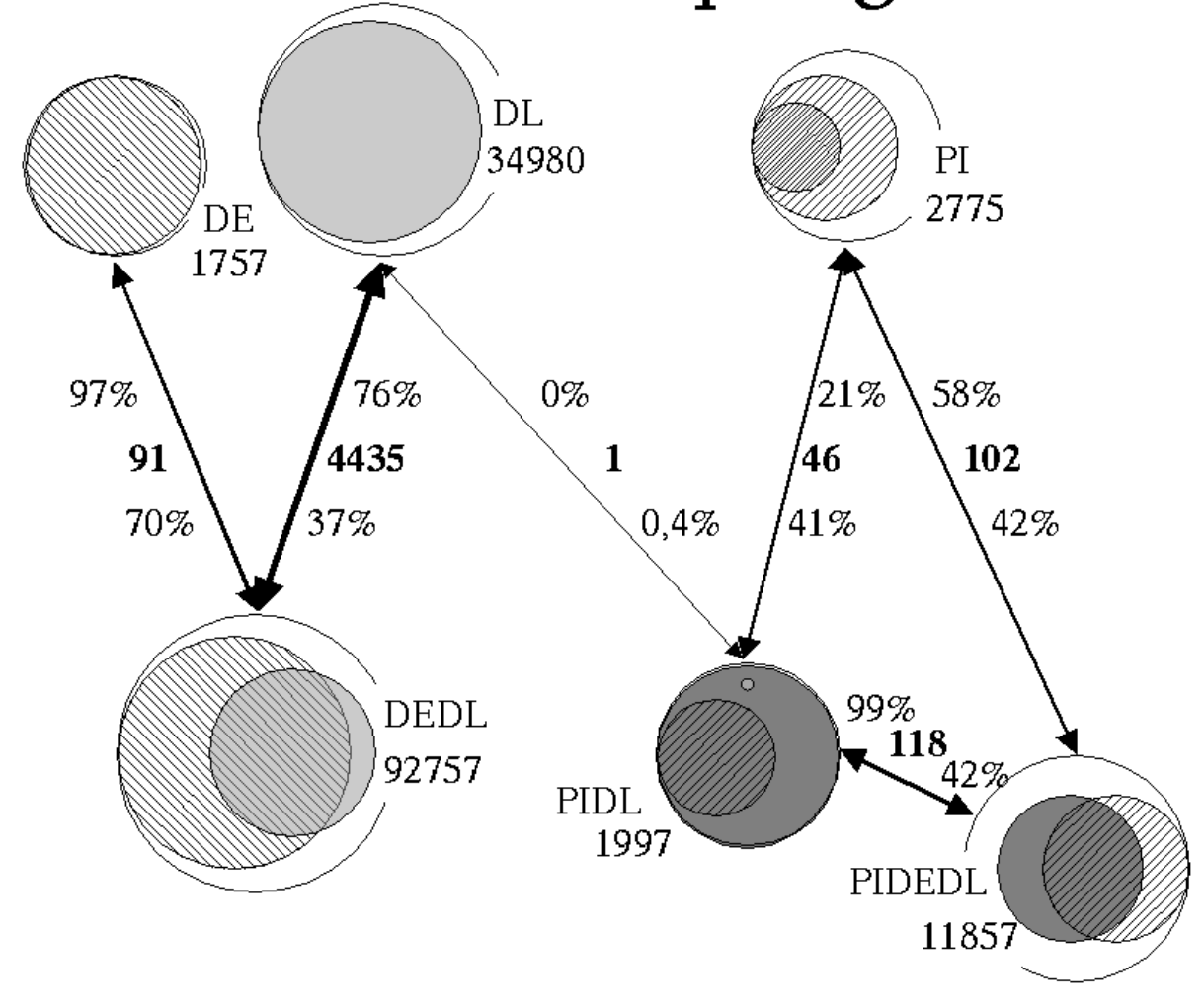

Beiträge von Deutsches Edelschwein $\bigcirc$ Deutsche Landrasse $\oslash$ Pietrain

Abb. 2: Anzahl der Eltern mit Nachkommen in zwei Genotypen sowie Anteil an Datensätzen der durch diese erklärt wird (Number of parents with offsprings in two genotyps and part of offsprings that has this animals as parents )

Modell für die Parameterschätzung

Als Modell wurde entsprechend der unterschiedlichen Genotypen ein Sechsmerkmalstiermodell genutzt, wobei die Leistungen in den unterschiedlichen genetischen Konstruktionen als jeweils separates Merkmal betrachtet wurden. Damit ist eine Abbildung unterschiedlicher genetischer Varianzen möglich, welche auch unterschiedliche genetische Korrelationen zwischen den Ausgangsrassen und den Kreuzungsprodukten beinhalten. Dagegen können jedoch weder unterschiedliche Beiträge der Elternlinien zur additiv genetischen Varianz in Kreuzung noch etwaige Dominaz- bzw. Epistatische Effekte abgebildet werden. Die Konsequenzen dieser Modellverletzungen werden detailliert bei SPILKE u.a. (1998) sowie MIELENZ u.a (2000) behandelt. Eine Umsetzung ist jedoch bisher mit allgemein verfügbaren Programmen zur Parameter- und Zuchtwertschätzung nicht möglich.

Das Modell stellt sich in Matrixschreibweise wie folgt dar:

$$
y=X b+Z a+P c+e
$$

mit $\quad y=$ Vektor der phänotypischen Beobachtungswerte 
$b=$ Vektor der fixen Effekte und Kovariablen

$a=$ Vektor der zufälligen additiv genetischen Effekte

$\mathrm{c}=$ Vektor der zufälligen Wurfumweleffekte

$e=$ Vektor der zufälligen Resteffekte

$X, Z$ und $P=$ Designmatritzen zur Zuordnung der jeweiligen Effekte zu den Beobachtungen

Erwartungswerte sind $E(y)=X b$ sowie $E(a)=E(c)=E(e)=0$. Die VarianzKovarianzmatrix der zufälligen Effekte läßt sich wie folgt darstellen:

$$
\operatorname{var}\left[\begin{array}{l}
a \\
c \\
e
\end{array}\right]=\left[\begin{array}{ccc}
A \otimes \sigma_{a}^{2} & 0 & 0 \\
0 & I \otimes \sigma_{c}^{2} & 0 \\
0 & 0 & I \otimes \sigma_{e}^{2}
\end{array}\right]
$$

mit $A=$ additiver Verwandtschaftsmatrix, $I=$ Einheitsmatrix, $\otimes=$ Kroneckerprodukt sowie $\sigma_{a}^{2}=$ Varianz der additiv genetischen Effekte sowie $\sigma_{c}^{2}=$ Varianz der zufälligen Wurfumwelteffeke und $\sigma_{e}^{2}=$ Varianz der zufälligen Resteffekte. Im Detail wird für die Zuchtwerte die folgende Varianz-Kovarianzmatrix unterstellt:

$$
\operatorname{var}(a)=\operatorname{var}\left[\begin{array}{c}
a_{11} \\
a_{22} \\
a_{33} \\
a_{44} \\
a_{55} \\
a_{66}
\end{array}\right]=\left[\begin{array}{cccccc}
A \otimes \sigma_{11}^{2} & A \otimes \sigma_{12} & A \otimes \sigma_{13} & A \otimes \sigma_{14} & A \otimes \sigma_{15} & A \otimes \sigma_{16} \\
& A \otimes \sigma_{22}^{2} & A \otimes \sigma_{23} & A \otimes \sigma_{24} & A \otimes \sigma_{25} & A \otimes \sigma_{26} \\
& & A \otimes \sigma_{33}^{2} & A \otimes \sigma_{34} & A \otimes \sigma_{35} & A \otimes \sigma_{36} \\
& & & A \otimes \sigma_{44}^{2} & A \otimes \sigma_{45} & A \otimes \sigma_{46} \\
& & & & A \otimes \sigma_{55}^{2} & A \otimes \sigma_{56} \\
\text { sym } & & & & & A \otimes \sigma_{66}^{2}
\end{array}\right]
$$

wie vorher sowie $\sigma_{i i}^{2}=$ Varianz der additiv genetischen Effekte und $\sigma_{i j}=$ Covarianz zwischen den additiv genetischen Effekten. Wobei die Indices 1, 2 sowie 4 die Reinzuchten kennzeichnen und 3, 5und 6 die entsprechenden Kreuzungsprodukte.

Als fixe Efefkte sind ein kombinierter Betriebs-Jahres-Monatseffekt und ein Geschlechtseffekt enthalten. Die Jahres-Monatseffekte wurden bei ausreichender Klassenbesetzung monatlich definiert. Bei ungenügender Klassenbesetzung $(<6$ Beobachtungen) wurden diese Klassen innerhalb des selben Betriebes der zeitlich am nächsten liegenden Klasse zugeschlagen. Die Tiere, die auf Station geprüft wurden, sind mit einer Stationskennzeichnung und nicht mit dem Herkunftsbetrieb codiert worden. Neben dem additiv genetischen Tiereffekt ist eine weitere zufällige Einflußgröße, ein permanenter Umwelteffekt enthalten, welcher als Wurfgeschwistereffekt definiert wurde. Letzterer ist für alle Tiere aus einem Wurf identisch.

In einem entsprechenden Sechsmerkmalsmodell für die Berücksichtigung der Leistungen in einem Dreirassenkreuzungsprogramm können formal 36 genetische Effekte definiert werden, wobei nur ein Teil dieser sinnvoll ist. Eine Schätzung der Zuchtwerte der Reinzuchttiere in Kreuzung erfolgt dann aus den Kreuzungsinformationen unter Berücksichtigung der genetischen Beziehungen zwischen Reinzucht und Kreuzungsleistungen und deren Beziehung zu den Zuchtwerten der Kreuzungstiere. Hierfür ist dann die Kenntnis der Varianzen in Reinzucht und Kreuzung sowie die der Kovarianzen zwischen diesen Leistungen notwendig.

Die Parameterschätzung wurde mittels REML-Methode nach dem Sechsmerkmalstiermodell mit dem Programmpaket VCE 4.2 (GROENEVELD, 1994) durchgefürt. 
3. $\quad$ Ergebnisse und Diskussion

In der folgenden Tabelle 2 sind die Schätzwerte für die Heritabilitäten (Diagonale) und die genetischen Korrelationen (oberhalb der Diagonale) zwischen den Genotypen dargestellt. Die nach Interpretierbarkeit sowie Datenumfang und Standardfehler relevanten Werte sind dabei hervorgehoben.

\section{Tabelle 2}

Ergebnisse der Parameterschätzung für das Merkmal Lebenstagszunahme: Heritabilitäten in der Diagonalen und genetische Korrelationen zwischen den Genotypen oberhalb der Diagonalen, der Standardfehler befindet sich jeweils unter dem Schätzer in Klammern (Estimated parameters for daily gain, heritabilities diagonal and genetic correlations above diagonal with standard errors of the ratios in brackets)

\begin{tabular}{cccccc}
\hline $\mathbf{D E}$ & $\mathbf{D L}$ & DEDL & PI & PIDL & PIDEDL \\
\hline $\mathbf{0 , 2 6}$ & 0,53 & $\mathbf{0 , 8 9}$ & 0,38 & 0,38 & 0,60 \\
$(0,04)$ & $(0,08)$ & $(0,04)$ & $(0,12)$ & $(0,17)$ & $(0,10)$ \\
& $\mathbf{0 , 3 1}$ & $\mathbf{0 , 8 6}$ & 0,67 & $\mathbf{0 , 7 7}$ & 0,69 \\
& $(0,01)$ & $(0,02)$ & $(0,09)$ & $(0,16)$ & $(0,08)$ \\
& & 0,61 & 0,65 & $\mathbf{0 , 7 4}$ \\
& & $(0,27$ & $0,11)$ & $(0,17)$ & $(0,09)$ \\
& & & $\mathbf{0 , 1 7}$ & $\mathbf{0 , 7 2}$ & $\mathbf{0 , 7 4}$ \\
& & $(0,04)$ & $(0,13)$ & $(0,04)$ \\
& & & $\mathbf{0 , 2 0}$ & $\mathbf{0 , 9 5}$ \\
& & & $(0,03)$ & $(0,02)$ \\
& & & & $\mathbf{0 , 2 4}$ \\
& & & & $(0,02)$ \\
\hline
\end{tabular}

Die Heritabilitäten in den einzelnen Genotypen schwanken zwischen 0,17 und 0,31 bei Berücksichtigung der Wurfumwelteffekte, welche zwischen 10 und $20 \%$ die Gesamtvariation erklären (bei einem Standardfehler von 0,003 bis 0,023).

In der Literatur erreichen die Heritabilitätsschätzungen bei Einbeziehung von Wurfumwelteffekten meistens ebenfalls Werte zwischen 0,1 und 0,3 bei vergleichbaren $c^{2}$-Schätzern zwischen 0,1 und 0,2 (z.B. THOLEN, 1990; GROENEVELD, 1991; BRANDT, 1994; SCHMUTZ, 1995; WOLF u.a., 1997). Höhere Heritabilitäten $(0,3-0,5)$ bei etwa gleichen Wurfumweltvarianzanteilen finden WILLMS u.a. (1998) bei der Untersuchung heterogener Varianzen auf unterschiedlichen Betrieben. Wesentlich höhere Schätzungen für den Wurfumwelteffekt $(0,4$ und 0,5) finden sich bei HOFER (1990), wobei er ähnliche $h^{2}$-Werte $(0,2)$ ermittelt. Diese hohen Werte führt er auf die häufig gleichzeitige Prüfung von Wurfgeschwistern im Feld zurück.

Die genetischen Korrelationen zwischen den Mutterrassen sind sehr hoch und erreichen Werte von etwa 0,9. Eine Betrachtung als separate Merkmale aufgrund einer unterschiedlichen genetischen Bedingtheit erscheint hier somit nicht notwendig. Anders dagegen bei der Kombination Pietrain mit den entsprechenden Kreuzungs-

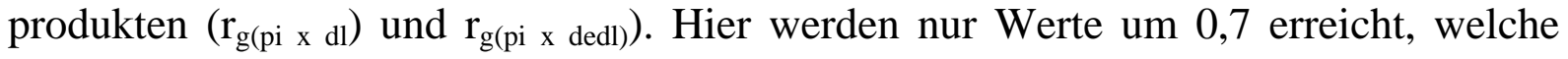
jedoch zu beiden Kreuzungsgenotypen sehr gleichmäßig sind.

Die genetischen Korrelationen zwischen den beiden mit Pietrain angepaarten Endprodukte liegt bei diesem Merkmal bei 0,95. Eine Zusammenfassung dieser Leistungen in einer Zuchtwertschätzung ist also möglich.

Geringer fallen die genetischen Korrelationen erwartungsgemäß zwischen den Ausgangsrassen aus $(0,53)$. Diese Korrelationen führen in einer Umsetzung dieses Modells in einer Zuchtwertschätzung dann beispielsweise auch zu Zuchtwerten von 
Landrassetieren, die sie in Reinzucht als Edelschwein erreichen würden. Auch aus diesem Grund ist hier eine Betrachtung als Fünfmerkmalsmodell derzeit nicht notwendig.

Literaturparameter für die genetischen Korrelationen für dieses Merkmal sind in der Tabelle 3 aufgeführt.

Tabelle 3

Literaturparameter für genetische Korrelationen zwischen Reinzucht- und Kreuzungsleistungen für Merkmale der Zunahmeleistung (ltz - Lebenstagszunahme; ptz - Prüftagszunahme) (Estimates of genetic correlations between purebreed and crossbred for daily gain in pigs (ltz- daily gain lifetime; ptz - daily gain in test station)

\begin{tabular}{llc}
\hline Zunahmeleistung & & \\
\hline BRANDT (1994) & ltz & $0,87-0,97$ \\
SCHMUTZ (1995) & ptz & 0,73 \\
TRAPPMANN und KIRSTGEN (1995) & ptz & 0,66 \\
BRANDT und TÄUBERT (1998) & ptz & 0,90 \\
FISCHER (1998) & ltz & 0,93 und 0,97 \\
\hline
\end{tabular}

Diese Parameter sind ausnahmslos hoch und stimmen mit den eigenen Schätzungen für die reine Einfachkreuzung wie auch für die direkten benachbarten Kreuzungsstufen $(0,72-0,95)$ überein.

4. Schlussfolgerungen

Aufgrund der genetischen Zusammenhänge zwischen den geprüften Genotypen im Merkmal Lebenstagszunahme ist für die Ermittlung von Reinzucht- und Kreuzungszuchtwerten für die Pietraineber ein Dreimerkmalsmodell mit folgender Merkmalsdefinition zu empfehlen:

- Leistungen der Mutterrassen und deren Einfachkreuzungen (DE, DL, DEDL)

Diese Zusammenfassung ist möglich, da die genetischen Korrelationen zwischen den Ausgangsrassen und der Kreuzung um 0,9 liegen. Weiterhin sind diese Korrelationen zu den Endprodukten der Dreirassenkreuzung sehr ähnlich $(0,6 ; 0,7 ; 0,7)$.

- Leistungen aller mit Pietrainvätern angepaarten Kreuzungsprodukte

Eine Zusammenfassung ist möglich da die genetischen Korrelationen zwischen den beiden hier untersuchten Genotypen (PIDL, PIDEDL) mit 0,95 fast Eins erreichen. Es sind also keine additiv genetisch bedingten Unterschiede zu erkennen.

- Leistungen der Pietraintiere

Diese getrennte Berücksichtigung ist zu empfehlen, da die genetischen Korrelationen zu den Kreuzungsprodukten mit Pietrainvätern jeweils nur 0,7 erreichen. Diese Größenordnung spricht aus genetischer Sicht für eine separate Formulierung.

Mit dieser Vorgehensweise wird die Leistung der Anpaarungsgrundlage auf die Leistung der Endprodukte angerechnet und somit korrigiert. Dabei sind jedoch neben den Vorteilen der gemeinsamen Schätzung von Reinzucht- und Kreuzungszuchtwerten in einem Modell die Nachteile der Nichtberücksichtigung der genetischen Korrelationen $\mathrm{zu}$ anderen Merkmalen innerhalb eines Genotypes gegeben. Für weitere 
Merkmale können aufgrund des derzeitig vorliegenden Materialumfanges keine ausreichend sicheren Schätzungen für die genetischen Parameter vorgelegt werden.

\section{Literatur}

BÖSCH, M.:

Die Selektion auf Wurfgröße beim Schwein unter besonderer Berücksichtigung der genetischen Beziehung zwischen Reinzucht- und Kreuzungsleistung. Univ. Kiel, Diss., 1999

BRANDT, H.:

Die Beziehung zwischen Produktionsmerkmalen von Reinzucht- und Kreuzungsschweinen und Konsequenzen für die Optimierung der Selektion. Univ. Göttingen, Habilitationsschrift, 1994

BRANDT, H.; TÄUBERT, H.: 1998 zitiert nach BÖSCH (1999)

FISCHER, R.:

Schätzung genetischer Parameter für Reinzucht- und Kreuzungsleistungen beim Schwein. Univ. Halle, Diss., 1998

GROENEVELD, E.:

A reparameterization to improve numerical optimization in multivariate REML (co)variance component estimation. Genet. Sel. Evol. 26 (1994), 537-545

GROENEVELD, E.:

Simultaneous REML estimation of 60 covariance components in an animal model with missing values

HOFER, A.: using the downhill-simplex-algorithm. 42. Jahrestagung der EVT, Berlin (1991) Polykopie

Schätzung von Zuchtwerten feldgeprüfter Schweine mit einem Mehrmerkmals-Tiermodell. Zürich, Diss., 1990

KRIETER, J.:

Zuchtplanung beim Schwein. Univ. Kiel, Habilitationsschrift, 1992

MIELENZ, N.; SCHÜLER, L.; GROENEVELD, E.:

Simultane Zuchtwertschätzung mit Reinzucht- und Kreuzungsleistungen unter Dominanz. Arch. Tierz., Dummerstorf 43 (2000), 87-98

RÖHE, R.; KRIETER, J.; PREISINGER, R.:

Bedeutung der Varianzkomponentenschätzung für die Zucht von landwirtschaftlichen Nutztieren - eine Übersicht. Arch. Tierz., Dummerstorf 43 (2000), 523-534

SCHMUTZ, M.:

Multivariate Schätzung von Populationsparametern für Merkmale aus Stations- und Feldprüfung der bayerischen Schweine-Herdbuchzucht in Reinzucht und Kreuzung. Univ. Kiel, Diss., 1995

SPILKE, J., GROENEVELD, E., MIELENZ, N.:

Joint purebred and crossbred (co)variance component estimation with a pseudo multiple trait model: loss in efficiency. J. Anim. Breed. Genet. 115 (1998), 341-350

THOLEN, E.:

Untersuchung von Ursachen und Auswirkungen heterogener Varianzen der Indexmerkmale in der Deutschen Schweineherdbuchzucht. Univ. Göttingen, Diss., 1990

TRAPPMANN, W.; KIRSTGEN, B.:

Genotyp-Umwelt-Interaktionen in Kreuzungszuchtprogrammen beim Schwein. 70. Sitzung des WEI, M.: genetisch-statistischen Ausschusses der DGfZ (1995) Polykopie

Combined crossbred and purebred selection in animal breeding. Wageningen, Habilitationsschrift, 1992

WILLMS, F.; RÖHE, R.; TIMM, H. H.; KALM, E.:

Schätzung genetischer Parameter für die Mutterlinien Large White und Landrasse unter Berücksichtigung unterschiedlicher Prüfumwelten. Züchtungskunde 70 (1998), 338-350

WOLF, J.; GROENEVELD, E.; WOLFOVA, M; JELENKOVA, V.:

Stand der Vorbereitungen zur Einführung des Mehrmerkmals-Tiermodells in der tschechischen Schweinezucht. Zusammenkunft der Spezialisten für Zuchtwertschätzung beim Schwein, Sächsische Landesanstalt für Landwirtschaft, Köllitsch (1997) Polykopie

Eingegangen: 24.06.2002

Akzeptiert: 20.08.2002

Anschriften der Verfasser

Dr. RALF FISCHER, Dr. ULF MÜLLER, Dr. UWE BERGFELD

Sächsische Landesanstalt für Landwirtschaft, Fachbereich für Tierzucht,

Fischerei und Grünland

Am Park 3

D-04886 Köllitsch 